\title{
A Comparative Study of the Short-Term Efficacy of Laparoscopic Radical Resection of Right-Sided Colon Cancer with Two Different Surgeon Positions and Trocar Placements
}

\author{
Ziling Zheng*, Maocai Tang*, Shouru Zhang\#, Hao Sun, Jingkun Shang \\ Department of Gastrointestinal Cancer Center, Chongqing University Cancer Hospital, Chongqing, China \\ Email: "zsrhjp@126.com
}

How to cite this paper: Zheng, Z.L., Tang, M.C., Zhang, S.R., Sun, H. and Shang, J.K. (2022) A Comparative Study of the ShortTerm Efficacy of Laparoscopic Radical Resection of Right-Sided Colon Cancer with Two Different Surgeon Positions and Trocar Placements. Journal of Cancer Therapy, 13, 105-116.

https://doi.org/10.4236/jct.2022.133008

Received: February 14, 2022

Accepted: February 28, 2022

Published: March 3, 2022

Copyright $\odot 2022$ by author(s) and Scientific Research Publishing Inc. This work is licensed under the Creative Commons Attribution International License (CC BY 4.0).

http://creativecommons.org/licenses/by/4.0/ (c) (i) Open Access

\begin{abstract}
Objective: To investigate the short-term efficacy of laparoscopic radical resection of right-sided colon cancer with two different surgeon positions and trocar placements. Methods: The data of 78 patients who underwent laparoscopic radical resection of right-sided colon cancer between January 2018 and August 2019 were retrospectively analysed. The surgical method was selected by the patients. The patients were divided into two groups according to the surgeons' positioning habits and trocar placements. The group with the lead surgeon standing between the patient's legs had 35 patients, and the group with the lead surgeon standing at the left side of the patient had 43 patients. The operation time, intraoperative blood loss, postoperative anal gas evacuation time, postoperative urinary catheter indwelling time, postoperative hospital stay, C-reactive protein (CRP) level on the first day after surgery, and postoperative pathological data and complications were compared between the two groups. Results: All patients underwent the laparoscopic radical resection of right-sided colon cancer, none converting to laparotomy. No significant difference $(\mathrm{P}>0.05)$ in intraoperative blood loss $(57.6 \pm 21.3 \mathrm{ml}$ vs $60.2 \pm 35.3 \mathrm{ml})$, postoperative anal gas evacuation time $(3.5 \pm 1.1 \mathrm{~d}$ vs $3.8 \pm$ $1.3 \mathrm{~d}$ ), postoperative urinary catheter indwelling time ( $2.6 \pm 1.3 \mathrm{~d}$ vs $2.4 \pm 1.2$ d), postoperative hospital stay ( $7.1 \pm 1.8 \mathrm{~d}$ vs $7.5 \pm 2.1 \mathrm{~d})$, or CRP level on the first day after surgery $(54.7 \pm 9.6 \mathrm{mg} / \mathrm{L}$ vs $53.9 \pm 8.2 \mathrm{mg} / \mathrm{L})$ was detected between the two groups. The operation time was shorter in the group with the lead surgeon standing between the patient's legs $(185.2 \pm 25.6 \mathrm{~min}$ vs $196.2 \pm$
\end{abstract}

*Ziling Zheng and Maocai Tang contributed equally to this work.

"Corresponding author. 
$19.7 \mathrm{~min})(\mathrm{P}<0.05)$. The two groups did not differ significantly in the tumour length $(4.2 \pm 1.3 \mathrm{~cm}$ vs $3.9 \pm 1.5 \mathrm{~cm})$, number of dissected lymph nodes (27.5 \pm 11.6 vs $25.1 \pm 15.4)$, pathological type, or postoperative pathological tumour-node-metastasis stage $(\mathrm{P}>0.05)$. No patients died or had anastomotic fistula during their postoperative hospital stay, and the incidence of postoperative complications did not differ between the two groups $(22.9 \%$ (8/35) vs $23.3 \%$ (10/42); P > 0.05). Conclusion: Under the principle of radical resection, the surgeon should adopt the most suitable standing position and trocar placement according to the specific situation. If the surgeon stands between the patient's legs, this might shorten the operation time and promote a smoother surgery.

\section{Keywords}

Laparoscopic Radical Resection, Right-Sided Colon Cancer, Surgeon

Positions, Trocar Placements

\section{Introduction}

Right-sided colon cancer is a common malignant tumour of the digestive tract and has a higher mortality rate than other colon cancers for multiple reasons, such as genetics, eating habits, and living habits. The incidence rate of right-sided colon cancer has been increasing in recent years [1]. Surgical resection is still the main treatment for right-sided colon cancer. With the popularization of laparoscopic surgery and the adoption of the concept of complete mesocolectomy, the resection scope of right-sided colon cancer surgery has been further standardized. Laparoscopic surgery can achieve the same radical therapeutic effect as laparotomy and has the advantages of smaller incisions and faster recovery times [2] [3].

The surgical technique of laparoscopic radical resection of right-sided colon cancer has matured. Even so, some details remain to be standardized, such as the standing position of the surgeon and the trocar placement location. This study collected the data of patients who underwent laparoscopic radical resection of right-sided colon cancer in our department between January 2018 and August 2019 and compared the safety, radical therapeutic effect, and short-term efficacy between two different surgeon positions and trocar placements.

\section{Materials and Methods}

\subsection{General Information}

The data of 78 patients who underwent laparoscopic radical resection of rightsided colon cancer in the Department of Gastrointestinal Surgery of Chongqing University Cancer Hospital between January 2018 and August 2019 were collected. The patients were divided into two groups according to the surgeons' positioning habits and trocar placements. The group with the lead surgeon stand- 
ing between the patient's legs had 35 patients, and the group with the lead surgeon standing at the left side of the patient had 43 patients. There were 47 were males and 31 females, with an age range of $32-76$ years. All patients underwent routine colonoscopic biopsy, contrast-enhanced chest-abdomen-pelvis computed tomography and serum tumour marker monitoring. The diagnosis was confirmed as right-sided colon cancer, including 21 hepatic flexure cancers and 57 ascending colon cancers. All patients successfully completed the surgery, and no patients were converted to laparotomy.

Inclusion criteria were as follows: 1) Patients were confirmed to have colon cancer through colonoscopic biopsy before surgery, and the lesions were in the right colon; 2) Contrast-enhanced chest-abdomen-pelvis computed tomography confirmed that the lesions were in the right colon resection region, with no invasion in the stomach, duodenum, kidney, ureter, psoas major muscle, or other surrounding organs; 3) Patients did not have distant metastasis in the liver, lung, or other distant organs; 4) Patients did not undergo neoadjuvant radiotherapy or chemotherapy before the surgery. Exclusion criteria were as follows: 1) Multiorigin colorectal cancer; 2) Unrelieved intestinal obstruction before the surgery; 3) Organ dysfunction, including of the heart, brain, or lungs, and non-tolerance of laparoscopic surgery; 4) Need for combined organ resection or emergency surgery. Each patient signed an informed consent form for laparoscopic surgery. The general information of two groups was not significantly different $(\mathrm{P}>0.05$; Table 1).

\subsection{Surgical Methods}

\subsubsection{Preoperative Preparation}

All surgeries were done by the same surgical team in our department, and the

Table 1. Characteristics of patients.

\begin{tabular}{|c|c|c|c|}
\hline Clinical factor & $\begin{array}{l}\text { Standing between } \\
\text { patient's legs } \\
(\mathrm{n}=35)\end{array}$ & $\begin{array}{l}\text { Standing at the left } \\
\text { side of patient } \\
(\mathrm{n}=43)\end{array}$ & $\begin{array}{c}\mathrm{P} \\
\text { value }\end{array}$ \\
\hline Age & $61.2 \pm 11.3$ & $59.5 \pm 12.6$ & 0.537 \\
\hline Male: Female & $21: 14$ & $26: 17$ & 0.967 \\
\hline $\operatorname{BMI}\left(\mathrm{kg} / \mathrm{m}^{2}\right)$ & $24.5 \pm 3.6$ & $25.1 \pm 4.2$ & 0.506 \\
\hline Previous abdominal surgery & 6 & 7 & 0.919 \\
\hline \multicolumn{4}{|l|}{ Tumor location } \\
\hline hepatic flexure & 9 & 12 & \multirow{2}{*}{0.828} \\
\hline Ascending colon & 26 & 31 & \\
\hline \multicolumn{4}{|l|}{ TNM staging before surgery } \\
\hline I & 6 & 7 & \\
\hline II & 16 & 20 & 0.994 \\
\hline III & 13 & 16 & \\
\hline
\end{tabular}


physicians in the team were highly experienced in laparoscopic surgery. Patients were given oral sodium phosphate solution on the night before surgery for routine bowel preparation and preventive intravenous antibiotics $30 \mathrm{~min}$ before the surgery. All operations were performed with urethral catheterization, endotracheal intubation, and general anaesthesia, and the STORZ high-definition laparoscopic surgery system was used.

\subsubsection{Surgeon Standing Position and Trocar Placement}

Patients were randomly divided into two groups, i.e., one group with the lead surgeon standing between the patient's legs and the other group with the lead surgeon standing at the left side of the patient. Trocars were placed according to the five-hole method. 1) Group with the lead surgeon standing between the patient's legs (Figure 1(A) and Figure 1(B)): The patient was in the supine splitleg position. A 12-mm trocar was placed in the abdominal cavity through a subumbilical incision to establish a $\mathrm{CO}_{2}$ pneumoperitoneum and make laparoscopic observations; a second $12-\mathrm{mm}$ trocar was placed at the inner $1 / 3$ of the connection between the left umbilicus and the anterior superior iliac spine, to be used as the main operation hole for the right hand of the lead surgeon; a 5-mm trocar was placed at the outer $1 / 3$ of the connection between the right umbilicus and the anterior superior iliac spine, to be used as the auxiliary operation hole for the left hand of the lead surgeon; a second 5-mm trocar was placed at the intersection between the left anterior axillary line and the upper horizontal line of the umbilicus, to be used as the operation hole for the left hand of the first assistant; and a third 5-mm trocar was placed $3 \mathrm{~cm}$ to the left of the midpoint of the line between the xiphoid process and umbilicus, to be used as the operation hole for the right hand of the first assistant. The lead surgeon stood between the patient's legs, the first assistant stood at the patient's left side, and the laparoscope holder stood outside of the patient's left leg. 2) Group with the lead surgeon standing at the left side of the patient (Figure 1(C) and Figure 1(D)): The patient was in the supine split-leg position. A $12 \mathrm{~mm}$ trocar was placed into the abdominal cavity through a longitudinal incision $3 \mathrm{~cm}$ under the umbilicus to establish a $\mathrm{CO}_{2}$ pneumoperitoneum and laparoscopic observation; a second $12 \mathrm{~mm}$ trocar was placed at $5 \mathrm{~cm}$ below the coastal margin of the left midclavicular line, which was used as the main operation hole for the right hand of the lead surgeon; a $5-\mathrm{mm}$ trocar was placed at the outer $1 / 3$ of the connection between the left umbilicus and the anterior superior iliac spine, to be used as the auxiliary operation hole for the left hand of the lead surgeon; a second $5-\mathrm{mm}$ trocar was placed $5 \mathrm{~cm}$ below the costal margin of the right midclavicular line, to be used as the auxiliary operation hole for the left hand of the first assistant; a third 5-mm trocar was placed at the outer $1 / 3$ of the connection between the right umbilicus and the anterior superior iliac spine, to be used as the auxiliary operation hole for the right hand of the first assistant. The lead surgeon stood on the left side of the patient, the first assistant stood on the right side of the patient, and the laparoscope holder stood between the patient's legs. 

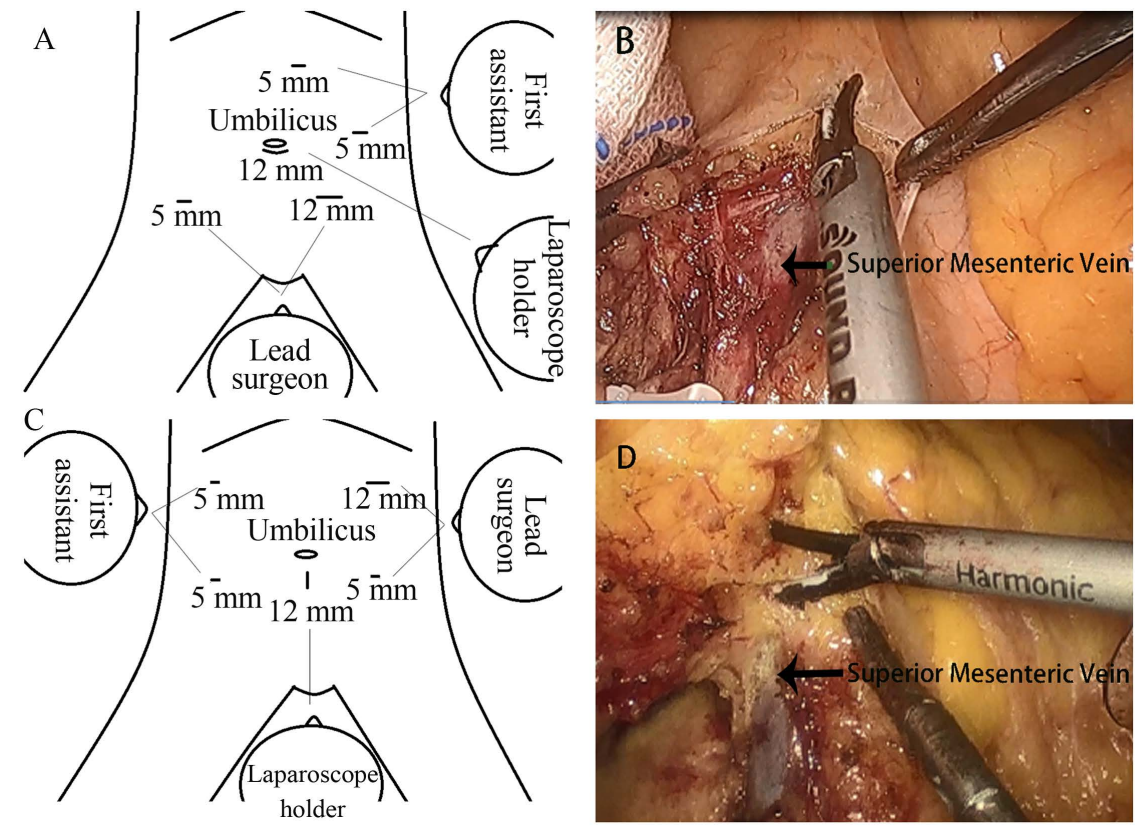

Figure 1. Laparoscopic radical resection of right-sided colon cancer with two different surgeon positions and trocar placements. (A) The schematic diagram with the lead surgeon standing between the patient's legs; (B) The surgical field with the lead surgeon standing between the patient's legs; (C) The schematic diagram with the lead surgeon standing at the left side of the patient; (D) The surgical field with the lead surgeon standing at the left side of the patient.

\subsubsection{Surgical Methods}

The traditional medial-to-lateral approach was adopted in both groups, and the principle of complete mesocolic excision was strictly followed. The surgical bed was tilted to the left. The small intestine was turned towards the left abdomen, and the protruded ileocolonic blood vessels were lifted with atraumatic forceps. The mesocolon underneath the blood vessels was cut open with an ultrasonic knife to enter the Toldt's space behind the right colon, and this plane was extended to the front and outside of the duodenum. The posterior ureter, reproductive vessels, and duodenum should be protected. The ileocolic artery and vein were first separated and exposed, then clamped and severed with a vascular clip at the root of the blood vessel. The posterior peritoneum was cut open along the surface of the superior mesenteric vein, revealing the arteries and veins of the right colon in sequence. The accessory right colon vein was clamped with a vascular clip and severed, and the lymphatic adipose tissue at the root of the blood vessel was dissected. The head of pancreas and duodenal ring was exposed. Damage to the pancreas and the main trunk of the mesenteric vessel should be prevented. The arteriovenous main trunk and right branch of the colon were exposed. The right arteriovenous branch of the colon in patients with ascending colon cancer was clamped with a vascular clip and severed, and the lymphatic adipose tissue at the root of the blood vessel was resected (the arteries in the colon of patients with hepatic flexure cancer should be ligated and severed at the root where the lymphatic adipose tissue was resected). Afterwards, the gastrocolic ligament 
of patients with ascending colon cancer was cut open along the outer edge of the vascular arch of the greater curvature of the stomach (it should be severed inside the vascular arch of the greater curvature of the stomach in patients with hepatic flexure cancer). The right gastroepiploic vessel was clamped and severed, and six lymph nodes underneath the pylorus were resected. In the omental sac, the hepatic flexure was isolated to the right along the hepatocolic ligament. Then, the ileocecal retroperitoneum was cut open to access the Toldt's space, and the lateral peritoneum was isolated from the ileocecum along the right paracolic sulci to the confluence of the hepatic flexure, followed by sharp separation of the inner side from the posterior end of the colon, until it met the isolated inner area. An incision on the right upper abdomen approximately $5 \mathrm{~cm}$ from the middle was made to enter the abdomen. The incision was protected with an incision protector, and the specimen was completely pulled out. The left $1 / 3$ of the transverse colon and the mesentery $15 \mathrm{~cm}$ from the ileocecum were cut open. The left $1 / 3$ of the transverse colon was cut open, the ileum $15 \mathrm{~cm}$ from the ileocecum was temporarily clamped, and the specimen was removed.

The abdominal wound was washed with a large amount of distilled water. A side-to-side anastomosis of the ileum and colon was made with a linear stapler. The anastomosis was examined to make sure there was no bleeding. The stump was closed with a linear stapler, and the anastomosis was stitched to stop bleeding. The incision was sealed to re-establish the pneumoperitoneum and flushed under laparoscopy. A groove negative-pressure drainage tube was placed at the right paracolic groove and fixed through the trocar hole at the right lower abdomen. After removing all materials used during the surgery, the incision was sutured layer by layer. After the surgery, the patients were treated with antibiotics and given parenteral nutrition support. The diet was gradually resumed. The drainage tube was removed after anal gas evacuation and defecation.

\subsection{Observation Indicators}

The operation time (from the beginning of skin incision to the end of suture), intraoperative blood loss (anaesthesia record), postoperative anal gas evacuation time, postoperative urinary catheter indwelling time, postoperative hospital stay, C-reactive protein (CRP) level on the first day after surgery, tumour length (the length of the intestinal tube invaded by the primary lesion on the postoperative pathology report), the number of resected lymph nodes, pathological type, and American Joint Committee on Cancer (AJCC) stage of the two groups were recorded and compared. Common perioperative complications, such as intestinal obstruction, abdominal haemorrhage, lung infection, incision infection, abdominal infection, chylous fistula, anastomotic fistula, and anastomotic bleeding, were recorded and analysed.

\subsection{Statistical Analysis}

All patients' clinical data were analysed with SPSS 17.0 software. Quantitative 
data are represented as mean \pm standard deviation $(\mathrm{x} \pm \mathrm{s})$ and analysed by the $\mathrm{T}$ test. Categorical data are represented as number and percentage and were analysed by the $\chi^{2}$ test. Differences with $\mathrm{P}<0.05$ were considered statistically significant.

\section{Results}

\subsection{Comparisons of Intraoperative and Postoperative Recovery}

All patients underwent laparoscopic right hemicolectomy and standard lymph node dissection, and no patients were converted to laparotomy. The two groups of patients did not differ significantly in intraoperative blood loss, postoperative anal gas evacuation time, postoperative urinary catheter indwelling time, postoperative hospital stay, or CRP level on the first day after surgery $(\mathrm{P}<0.05)$. The operation time of the group with the lead surgeon standing between the patient's legs was significantly shorter than that of the group with the lead surgeon standing at the left side of the patient $(\mathrm{P}<0.05$, Table 2$)$.

\subsection{Comparison of Postoperative Pathology and Complications}

All specimens were sent for pathological examination in accordance with standard regulations, and the lymph nodes were carefully sorted and counted by senior pathologists. The pathological results of the tumour resection margins of the two groups showed that there was no residual cancer, and the two groups did not differ significantly in tumour length, number of dissected lymph nodes, pathological types, or postoperative pathological tumour-node-metastasis (TNM) stage $(P>0.05$, Table 3 ). None of the patients in the two groups died or had anastomotic fistula during hospitalization. There were two cases of lung infection, one case of intestinal obstruction, one case of abdominal haemorrhage, two cases of incision infection, one case of abdominal infection, and one case of chylous fistula in the group with the lead surgeon standing between the patient's legs. The corresponding numbers in the group with the lead surgeon standing at the left side of the patient were one, two, zero, three, two, and two, respectively.

Table 2. Operative characteristics.

\begin{tabular}{cccc}
\hline Clinical factor & $\begin{array}{c}\text { Standing } \\
\text { between } \\
\text { patient's legs } \\
(\mathrm{n}=35)\end{array}$ & $\begin{array}{c}\text { Standing at } \\
\text { the left side } \\
\text { of patient } \\
(\mathrm{n}=43)\end{array}$ & $\begin{array}{c}\mathrm{P} \\
\text { value }\end{array}$ \\
\hline Operation time (min) & $185.2 \pm 25.6$ & $196.2 \pm 19.7$ & $\mathbf{0 . 0 3 5}$ \\
Intraoperative blood loss (ml) & $57.6 \pm 21.3$ & $60.2 \pm 35.3$ & 0.703 \\
Postoperative anal gas evacuation time (d) & $3.5 \pm 1.1$ & $3.8 \pm 1.3$ & 0.281 \\
Urinary catheter indwelling time (d) & $2.6 \pm 1.3$ & $2.4 \pm 1.2$ & 0.483 \\
Postoperative hospital stay (d) & $7.1 \pm 1.8$ & $7.5 \pm 2.1$ & 0.377 \\
Postoperative CRP level (mg/L) & $54.7 \pm 9.6$ & $53.9 \pm 8.2$ & 0.693 \\
\hline
\end{tabular}


Table 3. Characteristics of pathology and complications.

\begin{tabular}{cccc}
\hline Clinical factor & $\begin{array}{c}\text { Standing } \\
\text { between } \\
\text { patient's legs } \\
(\mathrm{n}=35)\end{array}$ & $\begin{array}{c}\text { Standing at } \\
\text { the left side } \\
\text { of patient } \\
(\mathrm{n}=43)\end{array}$ & $\begin{array}{c}\text { P } \\
\text { value }\end{array}$ \\
\hline Tumour length (cm) & $4.2 \pm 1.3$ & $3.9 \pm 1.5$ & 0.354 \\
Number of dissected lymph nodes (n) & $27.5 \pm 11.6$ & $25.1 \pm 15.4$ & 0.448 \\
Pathological type & 12 & 14 & \\
Uplift type & 14 & 19 & 0.761 \\
Ulcer type & 9 & 10 & \\
Invasive type & & & \\
I & 4 & 3 & \\
II & 15 & 18 & 0.761 \\
III & 16 & 22 & \\
Postoperative pathological TNM stage & 8 & 10 & \\
\hline
\end{tabular}

All patients were cured and discharged after symptomatic treatment, and no patients underwent unplanned surgery. The two groups did not differ significantly in the incidence of complications $(\mathrm{P}<0.05$, Table 3$)$.

\subsection{Follow-Up}

A total of 73 patients were followed up by outpatient visit or telephone, the follow-up time being 3 - 16 months with an average of 8 months. One patient died of multiple lung and liver metastases after 13 months. Five patients were lost to follow-up.

\section{Discussion}

With the continuous advancement of minimally invasive (laparoscopic) surgery in recent years, laparoscopic radical resection of right-sided colon cancer has become a conventional surgical treatment. The superiority of complete mesocolon resection has gained a consensus. The surgery requires sufficient intestinal resection scope and lymphatic dissection at the root of the blood vessel to obtain more dissected lymph nodes and improve the radical curative effect [4] [5]. The surgeon's familiarity with the normal anatomy and variations of the superior mesenteric vessels and their primary branches is the basis and the guarantee for successful laparoscopic radical resection of right-sided colon cancer [6]. The surgeon's choice of a suitable surgical approach and the use of skilled surgical techniques are the keys to the success of the surgery. Appropriate standing positions of the surgical team and trocar placement layout are extremely important for successful exposure and separation of various anatomical levels and harmo- 
nious cooperation among the team to complete the surgery. This study aimed to investigate the safety, the radical resection of the tumour, and the short-term efficacy of two different types of surgeon positions and trocar placements for the laparoscopic radical resection of right-sided colon cancer.

The laparoscopic radical resection of right-sided colon cancer is becoming more mature, and the scope of intestinal and mesangial resection and lymphatic dissection has been standardized and basically reached a consensus by the academic community. However, no consensus has been reached on the details of lymph node dissection and surgical approaches, which has become the focus of current debate [7] [8] [9]. There is no unified opinion on surgeon standing position or trocar placement, which are generally decided by observation convenience under laparoscopy, exposure of the primary lesions, cooperation between the lead surgeon and the assistants, separation and exposure of the lesions, complete mesangial and lymphatic dissection, and minimal secondary damage [10]. Following the principles of radical resection, surgeons often choose different standing positions and trocar placements based on their own experiences. Currently, most surgeons in the world adopt the five-hole medial-to-lateral approach for right colon resection and a trocar placement with the lead surgeon standing at the left side of the patient, while some surgeons adopt a trocar placement with the lead surgeon standing between the legs of the patient [11]. There have been no controlled studies on the standing positions of the surgeons and trocar placement. This study divided the patients into two groups according to the surgeon and trocar placements, with the lead surgeon standing between the patient's legs in one group and on the left side of the patient in the other group. Analysis of the most common complications after the radical resection of the right colon showed that no patients died or had anastomotic fistula during the postoperative hospital stay in either group, and the incidence of perioperative complications, such as lung infection, intestinal obstruction, abdominal haemorrhage, incision infection, abdominal infection, and chylous fistula, did not differ significantly between the two groups. The findings suggest that the safety of the surgery is fully guaranteed with the two surgeon standing positions and trocar placements, so both can be safely adopted in clinical practice.

To further compare the impact of the two surgeon standing positions and trocar placements on the radical treatment of tumour, the postoperative pathological data were analysed. There was no residual cancer at the tumour resection margins of either group, and the two groups did not differ significantly in tumour length, a number of dissected lymph nodes, postoperative pathological types, or postoperative pathological TNM stage, suggesting that both approaches can meet the requirements of radical colon cancer resection and meet the requirements of standardized surgery. Further analysis of relevant surgical indicators showed that the two groups did not differ significantly in intraoperative blood loss, postoperative anal gas evacuation time, postoperative urinary catheter indwelling time, postoperative hospital stay, or CRP level on the first day after surgery, but the operation time of the group with the lead surgeon standing 
between the patient's legs was significantly shorter than that of the group with the lead surgeon standing at the left side of the patient.

The two groups did not differ significantly in the safety or radical curative effect of the surgery, but the analysis of surgeons' experiences showed that when the lead surgeon stood between the patient's legs, the first assistant was on the left side of the patient and the laparoscope holder was on the outside of the patient's left leg. With the assurance of a correct view by the laparoscope holder, the assistant could lift the protruded ileocolonic blood vessel and the dorsal side of the middle mesentery of the transverse colon. Therefore, the field of view of the lead surgeon was perpendicular to the superior mesenteric blood vessel, and the main operating hole was in the patient's left lower abdomen. This made the operation of the ultrasonic knife more convenient and made it easier to anatomically expose the superior mesenteric blood vessel from the bottom up for thorough lymphatic adipose tissue dissection. Especially for obese patients, this positioning makes it easier to access the Toldt's space and then thoroughly dissect the lymphatic adipose tissue to the right of the superior mesenteric blood vessel (Figure 1(B)).

When the lead surgeon stood on the left side of the patient, the first assistant stood at the right side of the patient and the laparoscope holder stood between the patient's legs. In this way, although the observation view of the laparoscope holder was also perpendicular to the superior mesenteric blood vessel, the main operation hole of the lead surgeon was in the upper left abdomen of the patient, and the operation direction was from the horizontal direction left of the superior mesenteric blood vessel (Figure 1(D)), which was more time-consuming and made it harder to thoroughly dissect the lymphatic adipose tissue on the right of the superior mesenteric blood vessel than it was with the other way. As a result, the operation time of the group with the lead surgeon at the left side of the patient was longer than that of the other group, which increased the anaesthesia duration and the financial burden of the patient.

There are disadvantages to the lead surgeon standing between the patient's legs. Compared to the lead surgeons who stood at the left side of the patient, the lead surgeons who stood between the patient's legs found that it was difficult to sever the hepatocolic ligament and isolate the hepatic flexure, so they adopted the more sufficient lateral dissociation of the colon to make up for that limitation and shorten the operation time.

In summary, we believe that, due to the limited surgical field of view and the lack of the sense of distance and organ relationships in the laparoscopic radical resection of right-sided colon cancer, it is easy for the surgeon to enter the wrong anatomical level from the wrong viewing angle, resulting in unnecessary secondary injuries. Additionally, high laparoscopic proficiency of the surgeon and team cooperation is required, and the positioning habits of the surgeon and trocar placement layout are very important for surgical operation and cooperation among the surgical team. We believe that under the principle of radical 
treatment, the surgeon should adopt a more suitable standing position and trocar placement according to specific conditions. When the surgeon stands between the patient's legs, this can shorten some operations somewhat and promote a smoother surgery. Further investigation is required to determine the long-term postoperative efficacy in a larger sample size.

\section{Acknowledgements}

This study was supported by a Grant from Chongqing medical scientific research project (Joint project of Chongqing Health Commission and Science and Technology Bureau) (No. 2021MSXM309), and Chongqing Social Science Planning project (No. 2018BS47).

\section{Conflicts of Interest}

All the authors indicate no potential conflict of interest.

\section{References}

[1] Pochhammer, J., Köhler, J. and Schäffer, M. (2019) Colorectal Surgical Site Infections and Their Causative Pathogens: Differences between Left and Right-Side Resections. Surgical Infections, 20, 62-70. https://doi.org/10.1089/sur.2018.143

[2] Xie, D., Yu, C., Gao, C., et al. (2017) An Optimal Approach for Laparoscopic $\mathrm{D}_{3}$ Lymphadenectomy Plus Complete Mesocolic Excision $\left(\mathrm{D}_{3}+\mathrm{CME}\right)$ for Right-Sided Colon Cancer. Annals of Surgical Oncology, 24, 1312-1313. https://doi.org/10.1245/s10434-016-5722-1

[3] Benz, S.R., Tannapfel, A., Tam, Y., et al. (2015) Complete mesocolic excision for right-sided colon cancer-the role of central lymph nodes. Zentralblatt fur Chirurgie, 140, 449-452.

[4] Zurleni, T., Cassiano, A., Gjoni, E., et al. (2018) Surgical and Oncological Outcomes after Complete Mesocolic Excision in Right-Sided Colon Cancer Compared with Conventional Surgery: A Retrospective, Single-Institution Study. International Journal of Colorectal Disease, 33, 1-8. https://doi.org/10.1007/s00384-017-2917-2

[5] Ouyang, M., Luo, Z., Wu, J., et al. (2019) Comparison of Outcomes of Complete Mesocolic Excision with Conventional Radical Resection Performed by Laparoscopic Approach for Right Colon Cancer. Cancer Management and Research, 11, 8647-8656. https://doi.org/10.2147/CMAR.S203150

[6] Shapiro, R., Keler, U., Segev, L., et al. (2016) Laparoscopic Right Hemicolectomy with Intracorporeal Anastomosis: Short- and Long-Term Benefits in Comparison with Extracorporeal Anastomosis. Surgical Endoscopy, 30, 3823-3829. https://doi.org/10.1007/s00464-015-4684-x

[7] Dai, W., Zhang, J., Xiong, W., et al. (2018) Laparoscopic Right Hemicolectomy Oriented by Superior Mesenteric Artery for Right Colon Cancer: Efficacy Evaluation with a Match-Controlled Analysis. Cancer Management and Research, 10, 5157-5170. https://doi.org/10.2147/CMAR.S178148

[8] Sndenaa, K., Quirke, P., Hohenberger, W., et al. (2014) The Rationale Behind Complete Mesocolic Excision (CME) and a Central Vascular Ligation for Colon Cancer in Open and Laparoscopic Surgery: Proceedings of a Consensus Conference. International Journal of Colorectal Disease, 29, 419-428. https://doi.org/10.1007/s00384-013-1818-2 
[9] Siani, L.M. and Pulica, C. (2015) Laparoscopic Complete Mesocolic Excision with Central Vascular Ligation in Right Colon Cancer: Long-Term Oncologic Outcome between Mesocolic and Non-Mesocolic Planes of Surgery. Scandinavian Journal of Surgery, 104, 219-226. https://doi.org/10.1177/1457496914557017

[10] Brunner, M., Weber, G.F., Wiesmüller, F., et al. (2020) Laparoskopische Hemikolektomie Rechts Mit Kompletter Mesokolischer Exzision (CME). Zentralblatt Fur Chirurgie, 145, 17-23. https://doi.org/10.1055/a-1024-4629

[11] Rutgers, M. and Kahn, J. (2020) Comparing Standard Laparoscopic Hemicolectomy to CME Radical Right Colectomy for Patients with Right Sided Colon Cancer: A Randomized Controlled Feasibility Trial. European Journal of Surgical Oncology, 46, 2 p. https://doi.org/10.1016/j.ejso.2019.11.460 